Devel opment of Magnet i c Fi el d Cont rol for Magnet i cal I y Target ed Drug Del i very System Usi ng a Super conduct ing Nagnet

\begin{tabular}{|l|l|}
\hline 著者 & $\begin{array}{l}\text { M SH MA Fumi hi to, TAKEDA Shi n- i chi , I ZUM } \\
\text { Yoshi nobu, N SH JI MA Shi gehi ro }\end{array}$ \\
\hline $\begin{array}{l}\text { j our nal or } \\
\text { publ i cat i on ti t I e }\end{array}$ & I EEE transact i ons on appl i ed super conduct i vi ty \\
\hline vol une & 17 \\
\hline number & 2 \\
\hline page range & 2303 2306 \\
\hline year & $2007-02$ \\
\hline URL & ht t p: //hdl . handl e. net /10098/2394 \\
\hline
\end{tabular}




\title{
Development of Magnetic Field Control for Magnetically Targeted Drug Delivery System Using a Superconducting Magnet
}

\author{
Fumihito Mishima, Shin-ichi Takeda, Yoshinobu Izumi, and Shigehiro Nishijima
}

\begin{abstract}
We have been developing the device for magnetically targeted drug delivery system (MT-DDS), which can allow us to navigate and to accumulate the drug at the local diseased part inside the body by controlling to magnetic field strength and/or gradient generated by the superconducting magnets. In considering an application of this technique to the network of the blood vessel, a number of magnets should be placed within a small region. The magnetic field at the branching point is superimposed the fields from the magnets. The optimal arrangement of magnetic field for MT-DDS was calculated and experimental verification was made using several different sizes of Y-shaped glass tubes with multiple branching points as a model system of blood vessel. Ferromagnetic particles were injected into the $Y$-shape glass tube and were navigated by the magnetic field which was generated by the magnet optimally arranged based on the calculation. The ratio of the amount of the navigated ferromagnetic particle to the dosed amount was measured as magnetic navigation efficiency. It is found that experimental result of magnetic navigation efficiency agreed with the calculation results, which shows that magnetic navigation by the superconducting magnet can be a promising way for realization of MT-DDS.
\end{abstract}

Index Terms-Ferromagnetic particle, MT-DDS, navigation, superconducting magnets.

\section{INTRODUCTION}

$\mathbf{T}$ HE drug delivery system (DDS) that accurately transports the drug of the proper quantity to the diseased area can be expected to give a high efficiency of drug delivery and reduce the side effect. In addition, it can cure the diseased part located at the very difficult part to surgically approach and allow us to provide an effective treatment method to an obstinacy disease.

In the present study, emphasis was paid upon developing the application of the technology of the particle control by magnetic force. We have examined magnetic targeted DDS (MT-DDS) [1]-[3] which use magnetically seeded drug with the ferromagnetic particle (called 'Magnetic seeding drug', in following text) and magnetic force of the magnetic field which is generated outside of the body, carry the drug to the diseased part.

Analysis and an experiment were conducted in order to control the suspended magnetic particle inside the Y-shaped glass

Manuscript received August 28, 2006

The authors are with the Division of Sustainable Energy and Environmental Engineering, Graduate School of Engineering, Osaka University, 2-1 Ymada-oka, Suita, Osaka 565-0871, Japan (e-mail: f-mishima@qb.see.eng. osaka-u.ac.jp; stakeda@see.eng.osaka-u.ac.jp; izumi@see.eng.osaka-u.ac.jp; nishijima@see.eng.osaka-u.ac.jp).

Color versions of one or more of the figures in this paper are available online at http://ieeexplore.ieee.org.

Digital Object Identifier 10.1109/TASC.2007.898413

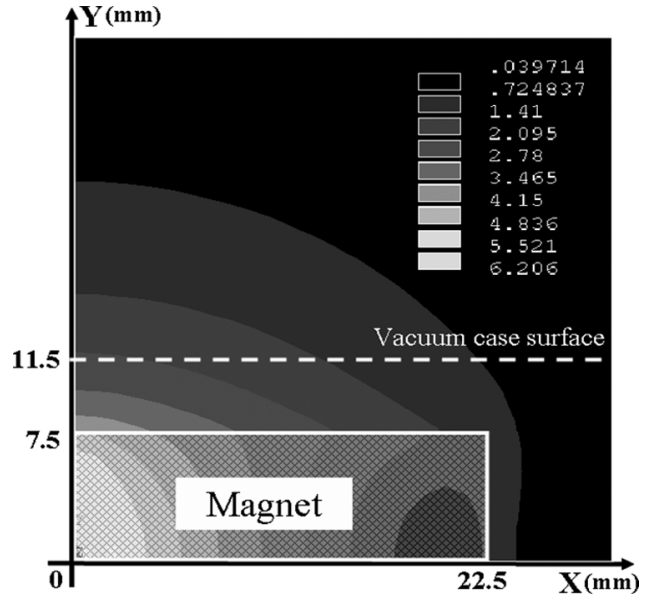

Fig. 1. Magnetic field analysis result, magnetic flux density B (white color represents strong magnetic flux density and black color represents weak one).

tube by magnetic force generated by the bulk superconductor magnet placed at some distant which imitated the blood vessel, and thus the accumulation and navigation of a flowing ferromagnetic particulate were tried and the feasibility of MT-DDS has been shown [2]-[5]. Furthermore, if navigation of the ferromagnetic drug can be carried out precisely at the branching point, the magnetic drug could be efficiently accumulated in a blood vessel of the living body.

The feasibility of magnetically control of the ferromagnetic particle in a Y-shaped blood vessel was examined using the bulk superconductor magnet. When we performed preliminary test where the magnet was merely placed near the branching point, ferromagnetic particulates were accumulated before a branching point and thus the navigation efficiency did not reach the satisfied level. Therefore, the novel navigation technique was developed at the present study by periodically changing the position of an externally-applied magnetic field and the obtained results were also reported.

\section{CAlculation Method}

\section{A. Analysis of Generated Magnetic Field and Flow Rate Distribution}

Calculated result of magnetic flux density of employed bulk superconducting magnet using ANSYS 9.0 was shown in Fig. 1. This analysis was performed under the condition where the electric current density was taken as the value of $3.8 \times 10^{8} \mathrm{~A} / \mathrm{m}^{2}$ and the right-upper part of the generated magnetic field was shown in this figure. 


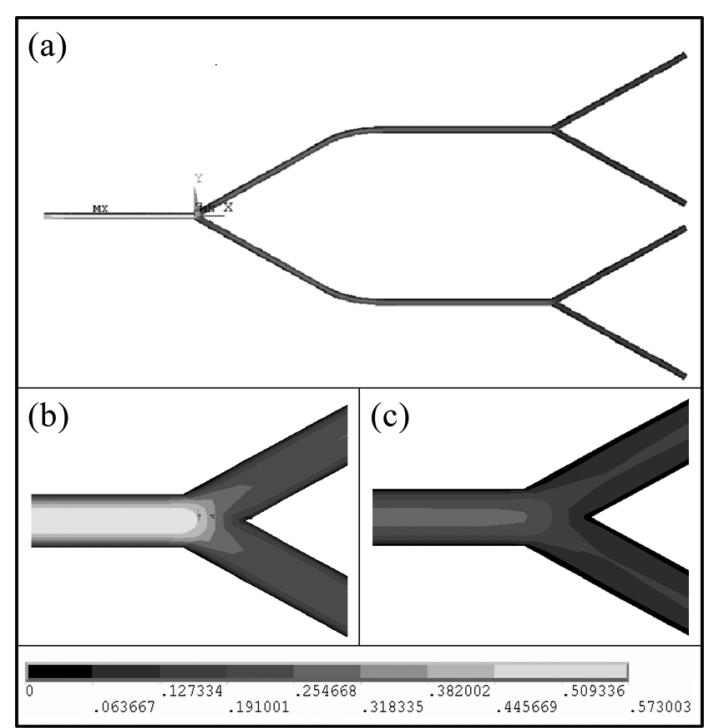

Fig. 2. (a) Contour plot of fluid velocity in vascular channel (b) Contour plot of fluid velocity in first branch of vascular channel (c) Contour plot of fluid velocity in second branch of vascular channel.

The flow rate distribution of Y-shaped blood vessel was calculated by the finite element method (FEM) using ANSYS (R) Release 9.0 (ANSYS, Inc.), showing in Fig. 2. In flow rate in the blood vessel the maximum values was taken as $50 \mathrm{~cm} / \mathrm{s}$ at the center of the vessel. The flow manner was a laminar flow and flow rate reached the maximum before the first branching point.

\section{B. Particle Trajectory Analysis}

The trajectory analysis of the ferromagnetic particles in vessel was performed in artery of human circulatory system ( $2 \mathrm{~mm}$ in diameter, about $40 \mathrm{~cm} / \mathrm{s}$ in mean flow velocity, $2.8 \mathrm{mPa} \cdot \mathrm{s}$ in fluid viscosity) using the $\mathrm{Y}$-shaped glass vessel with the branching point of 30-degrees angle, (the abdominal aorta of a small animal will be used when we try a targeting test using animal. Although the angle of branching point was not completely the same as $30(30 \times 2=60)$ degrees, the 30-degree of branching point is almost equivalent angle and one of the typical branching angle.) when a strong magnetic field was applied. The targeted site in human body is the capillary vessel of lungs. In this case, as the particle with a diameter less than $2 \mu \mathrm{m}$ can not be clogged, the ferromagnetic particle with a diameter of $2 \mu \mathrm{m}$ was adopted for the first trial, because large particle can be easily controlled by magnetic force. By considering the practical application, magnetic guidance/targeting of the particle with a diameter of $100 \mathrm{~nm}$ have been examined. Trajectory of the particles was calculated from the result of the fluid analysis as well as the magnetic field analysis [6], [7]. In Fig. 3, the ferromagnetic particles were uniformly arranged in the inlet part. The initial rate of the particle was assumed to be the rate of flow in coordinates of the particle. Trajectory of the particles was calculated according to the time development, and then the final position of the particles was classified into seven parts such as $\mathrm{A}_{1}, \mathrm{~B}_{1}, \mathrm{~B}_{2}, \mathrm{C}_{1}, \mathrm{C}_{2}, \mathrm{C}_{3}$, and $\mathrm{C}_{4}$ shown in Fig. 3 .

In this calculation, the particle trajectory was obtained for every 2.5 seconds from the starting point, and achievement of successful navigation was taken as the case that particles reach

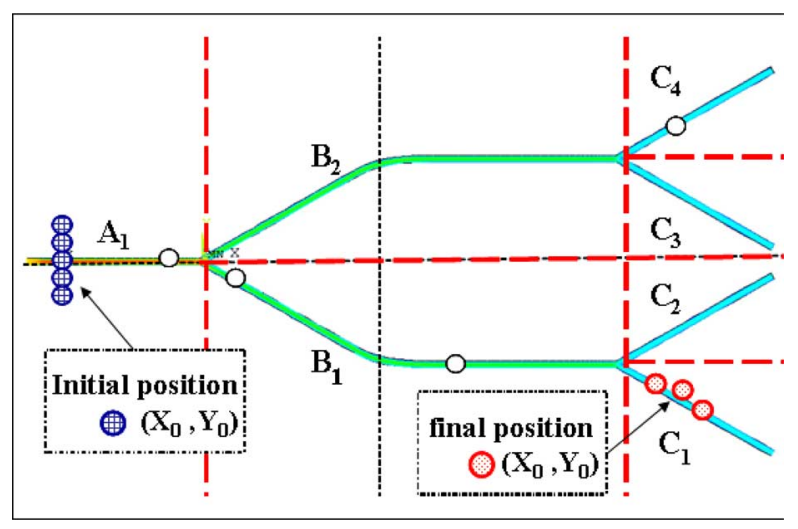

Fig. 3. Calculation of particle trajectory.

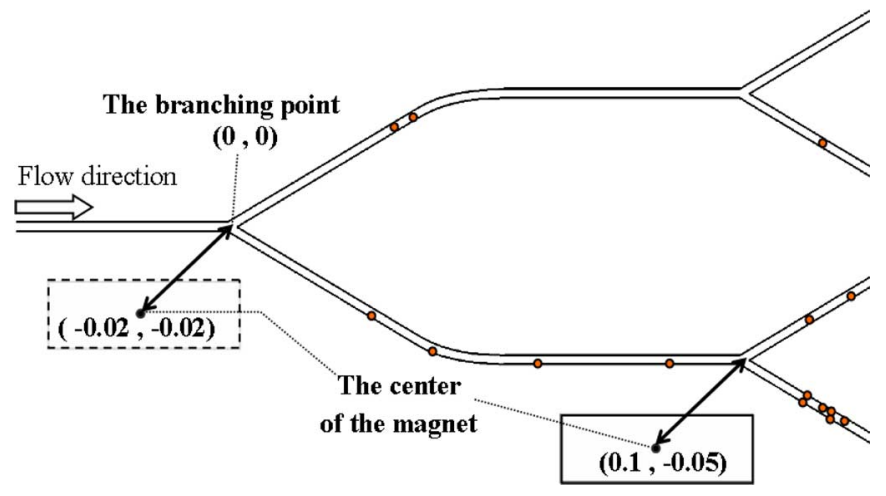

Fig. 4. Diagram illustrating trajectory of particles. [The system of coordinates with their origin $(0 \mathrm{~m}, 0 \mathrm{~m})$ at the center of the first branching point.]

to the area of $\mathrm{C}_{1}$. We also aimed to control the particles at all initial positions. The magnet which is a magnetic field center was fixed on $(-0.02,-0.02)$, when coordinate origin was set in branching point of fluid field. This condition was same as the first branching point and the second branching point.

Navigation efficiency was calculated when periodically changing the externally-applied magnetic field was applied. Fig. 4 shows calculation results of particle trajectory in condition where the magnet in first branching point was removed after 0.5 seconds from the calculation start, and magnet in second branching point was removed after 2.0 seconds.

\section{Calculation Results}

From this result, it was suggested that a novel method in which externally-applied magnetic field was periodically changed can provide the higher navigation efficiency to the targeted region. In this calculation condition, the distance between the first branching point and the second one was $14 \mathrm{~cm}$. And averaged flow rate in $B_{1}$ was assumed to be $20 \mathrm{~cm} / \mathrm{s}$, accumulated particles in first branching point can reach to the second point for 0.7 seconds. But it takes a little more time to reach because accumulated particles were stalled.

In a practical designing of MT-DDS, higher efficiency of navigation will be expected by applying magnetic field for longer time to pass between the first branching point and the second one. Additionally, when the particles are attracted from the generated magnetic field, it is necessary to consider the required 


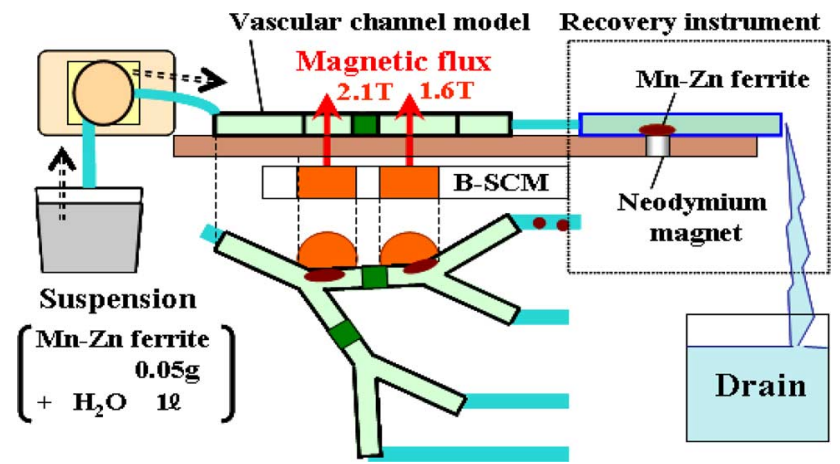

Fig. 5. Schematic illustration of experimental setup.

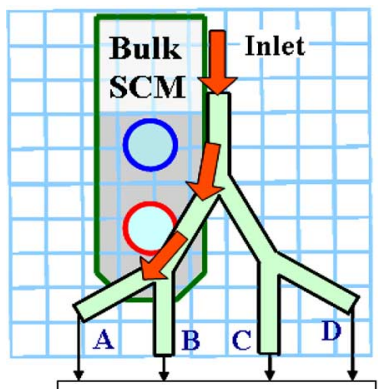

Recovery instrument

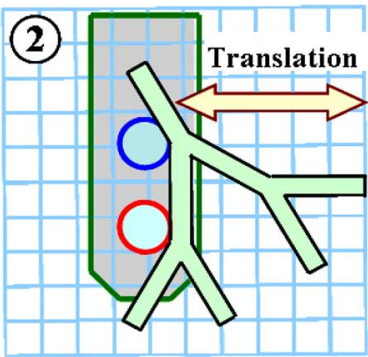

B-SCM:Bulk superconducting magnet

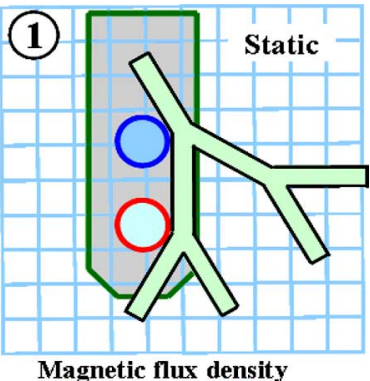

$2.1 \mathrm{~T}$

$1.6 \mathrm{~T}$

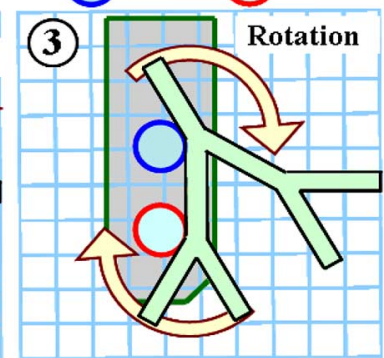

Fig. 6. Arrangement of bulk superconducting magnet.

time that a particle flows enough to be caught by magnetic field at the upper portion.

\section{EXPERIMENTAL PROCEDURE}

The schematic of an experimental apparatus as a vein model of the Y-shaped glass tubes using multiple bulk superconductor magnets was shown in Fig. 5. The Y-shaped glass tube with a diameter of $2 \mathrm{~mm}$ was placed above a bulk superconductor magnet.

Suspension of the ferromagnetic particles was flown in the vein model at the fixed flow rate $(40 \mathrm{~cm} / \mathrm{s})$ using the pump (EYELA RP-2000). Quantitative analysis of navigated ferromagnetic particle was made. These ferromagnetic particle employed in this study was a Mn-Zn ferrite particle (manufactured by Toda Kogyo) with the average particle diameter of $2 \mu \mathrm{m}$ and the saturation magnetization $0.33 \mathrm{~T}$.

In this experiment, the twin type of bulk superconducting magnet was used. The spatial arrangement of a twin type of bulk superconducting magnet [8], [9] and their periodic movement for magnetic navigation was shown in Fig. 6. In this experiment, navigation of ferromagnetic particle in the tube A in the Fig. 5

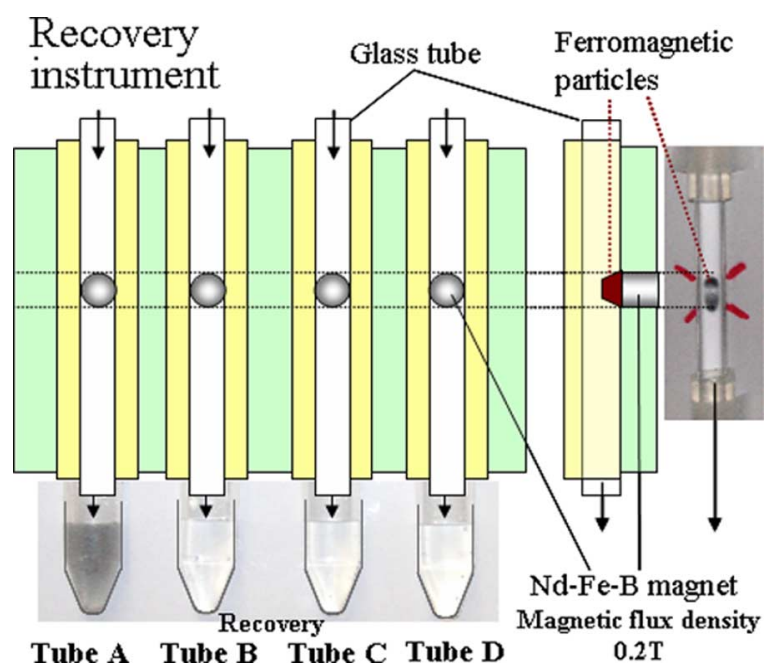

Fig. 7. Schematic illustration of recovery setup.

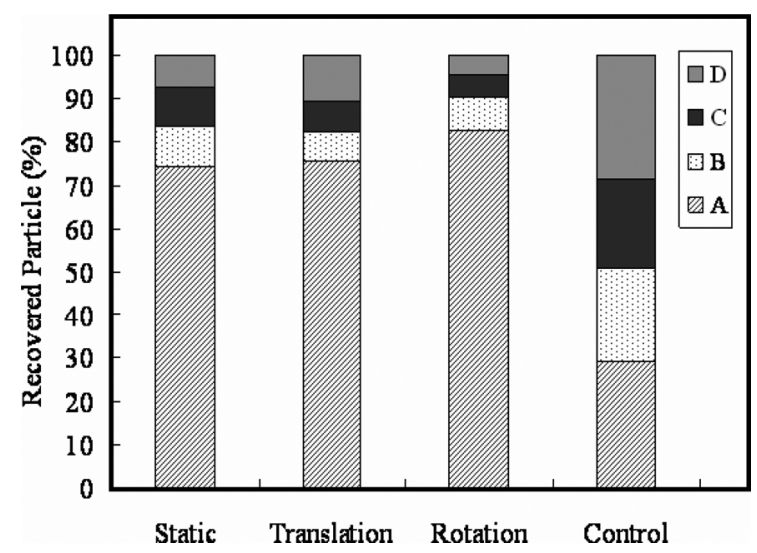

Fig. 8. Comparative diagram of $\mathrm{Fe}$ content.

was performed. The tested periodic movements were in three manners such as (1) static, (2) translational, and (3) rotational one, respectively.

The recovery system of ferromagnetic particles after navigation was shown in Fig. 7, in which neodymium magnet with surface-flux density $0.2 \mathrm{~T}$ was placed under the each tube to accumulate and recover the navigated magnetic particles.

\section{RESULTS AND DISCUSSION}

The quantity of navigated ferromagnetic particles was estimated using inductively coupled plasma-atomic emission spectroscopy (ICP-AES: SIMADZU, ICPS-7500) after completely dissolved to $\mathrm{Fe}$ ion in $\mathrm{HCl}$. The quantity of ferromagnetic particles without magnetic navigation was taken as a control. The navigation efficiency using different movement manner of the magnet was compared, showing in Fig. 8. From the result, when the magnets movement manner is horizontal to the flow channel and rotating manner around the middle point of them, higher navigation efficiency was seen than that in (1) static manner. Rotational transfer shows high efficiency more than $80 \%$ after two branching points. So it is concluded that (3) rotational manner of the magnet movement was the most suitable for navigation of the drug. 


\section{CONCLUSION}

From this experiment the feasibility of magnetically control of the ferromagnetic particles was confirmed in a blood vessel model consisting of Y-shaped glass tube and bulk superconducting magnets. This is a basic technology for MT-DDS that provides the drug navigation in the blood vessel of the circulatory organs system, which shows the usefulness of the medicine transportation methodology for MT-DDS.

In addition, the feasibility of efficient control to the intended direction was shown by the technique with periodic movement of magnetic field. The cases of moving the tube parallel to two magnets and rotating it centering on the middle of the two magnets, changing the magnetic field showed high navigation efficiency than the case of applying constant static magnetic field. This was proposed from the analysis and the experiment mentioned above, and proven by this experiment.

Especially, when the technique of parallel setup is compared with the rotation technique, in both of them an externally applying magnetic field was periodically decreased, and accumulating particles was navigated to the downstream. On this occasion it is thought that the parallel setup has a potential fault that an external applying magnetic field becomes a short period when the accumulated particles in the upstream reaches the downstream and in this respect the rotation technique was advantageous.

Therefore, the effectiveness of the technique was confirmed for navigating the accumulated particles on the tube wall at the part immediately before navigation by changing an external applying magnetic field at a constant cycle, and the effectiveness of the alternative cycle between back and forth of a divergence was proved by this experiment. In a word, the concept of setting some fictitious valves by an external applying magnetic field in each navigation of the circulatory organs system, and controlling the course of the magnetic particles in each navigation by the valve which opened and shut alternately, is a suitable external magnetic field applying technique for MT-DDS proposes by the main discourse.

As a result the feasibility of accomplishment of efficient navigation in an actual MT-DDS device design was suggested by calculating the time required for the particles to pass between the consecutive navigations from the average flow velocity there and distances between divergences and alternately impressing the magnetic field. In addition, it can be considered that it is necessary to regard the time which the particles once accumulated and put on the flow again required to flow up to the distance not caught by the external impressed magnetic field arranged by a divergence of the upstream.

\section{ACKNOWLEDGMENT}

The authors thank Prof. Hiroyuki Fujishiro (Iwate University, Faculty of Engineering) for allowing the use of the bulk superconducting magnet, many useful discussions, and support of this work.

\section{REFERENCES}

[1] M. O. Avilés, A. D. Ebner, H. Chen, A. J. Rosengart, M. D. Kaminski, and J. A. Ritter, "Theoretical analysis of a transdermal ferromagnetic implant for retention of magnetic drug carrier particles," Journal of Magnetism and Magnetic Materials, vol. 293, no. 1, pp. 605-615, May 2005.

[2] F. Mishima, S. Takeda, Y. Izumi, and S. Nishijima, "Three dimensional motion control system of ferromagnetic particle for magnetically targeted drug delivery system," IEEE Trans. Appl. Supercond., vol. 16, pp. 1539-1542, 2006.

[3] S. Takeda, F. Mishima, B. Terazono, Y. Izumi, and S. Nishijima, "Development of magnetic force-assisted new gene transfer system using biopolymer-coated ferromagnetic nanoparticles," IEEE Trans. Appl. Supercond., vol. 16, pp. 1543-1546, 2006.

[4] C. Alexiou, W. Arnold, R. J. Klein, F. G. Parak, P. Hulin, C. Bergemann, W. Erhardt, S. Wagenpfeil, and A. S. Lubbe, "Locoregional cancer treatment with magnetic drug targeting," Cancer Research, vol. 60, pp. 6641-6648, 2000.

[5] C. Alexiou, R. J. Schmid, R. Jurgons, M. Kremer, G. Wanner, C. Bergemann, E. Huenges, T. Nawroth, W. Arnold, and F. G. Parak, "Targeting cancer cell: Magnetic nanoparticles as drug carriers," Eur Biophys J., vol. 35, pp. 446-450, 2006.

[6] S. Fukui, Y. Takahashi, M. Yamaguchi, T. Sato, H. Imaizumi, M. Oizumi, S. Nishijima, and T. Watanabe, "Study on open gradient magnetic separation using superconducting solenoid magnet," IEEE Trans. Appl. Supercond., vol. 14, pp. 1568-1571, 2004.

[7] S. Fukui, M. Takahashi, T. Fujita, M. Yamaguchi, T. Sato, H. Imaizumi, M. Oizumi, S. Nishijima, and T. Watanabe, "Analytical study on open gradient magnetic separation using quadrupole magnetic field," IEEE Trans. Appl. Supercond., vol. 14, pp. 1572-1575, 2004.

[8] K. Yokoyama, M. Kaneyama, T. Oka, H. Fujishiro, and K. Noto, "Temperature measurement of RE123 bulk superconductors on magnetizing process," Physica C: Superconductivity, vol. 412-414, pt. 1, pp. 688-694, October 2004.

[9] H. Fujishiro, A. Fujiwara, T. Tateiwa, T. Oka, and H. Hayashi, "New type superconducting bulk magnet by pulse field magnetizing with usable surface on both sides in open space," IEEE Trans. Appl. Supercond., vol. 16, pp. 1080-1083, 2006. 